\title{
Intranasal Oxytocin Lessens the Attentional Bias to Adult Negative Faces: A Double Blind within-Subject Experiment
}

\author{
Youl-Ri Kim ${ }^{\circledR}$, Seung-Min Oh${ }^{1}$, Freya Corfield ${ }^{2}$, Da-Woon Jeong ${ }^{1}$, Eun-Young Jang ${ }^{1}$, and Janet Treasure ${ }^{2}$ \\ ${ }^{1}$ Department of Neuropsychiatry, Seoul Paik Hospital, Inje University, Seoul, Republic of Korea \\ 2Section of Eating Disorders, Department of Psychological Medicine, King's College London, Institute of Psychiatry, London, United Kingdom
}

\begin{abstract}
Objective Oxytocin is a neuropeptide that is involved in social emotional processing. A leading hypothesis is that oxytocin facilitates positive prosocial behaviors; the peptide may also play a more general role in inhibiting withdrawal-related social behaviors. The present study examined these possibilities.

Methods A double-blind, placebo controlled crossover design was used with 31 healthy women. Forty-five minutes following the administration of $40 \mathrm{IU}$ of intranasal oxytocin or a placebo, the participants were presented with two dot probe tests with pairs of face stimuli depicting emotional and neutral faces in adults.

Results Oxytocin specifically reduced the attention bias toward the location of the faces of adults showing negative emotions, particularly in the case of disgust. Oxytocin did not enhance the attentional bias toward adult happy faces. The effect of oxytocin toward adult negative emotion was correlated with the sensitivity of the drive in the behavioral motivational system.

Conclusion Oxytocin reduces attention to negative social emotions in adults, which supports oxytocin serves to inhibit withdrawalrelated social behaviour.

Psychiatry Investig 2014;11(2):160-166
\end{abstract}

Key Words Oxytocin, Emotion, Dot-probe task, Women.

\section{INTRODUCTION}

Oxytocin (OT) has been considered to be an important neuromodulatory hormone that is influential in parenting, particularly maternal behavior. ${ }^{1,2}$ Also, OT has been considered to be important in prosocial behavior between peers. ${ }^{3-6} \mathrm{~A}$ recent review of the influence of OT nasal spray on social cognition by Guastella and MacLeod ${ }^{7}$ concluded that overall OT enhances prosocial behavior between peers, but there are some inconsistent findings. ${ }^{8}$ One explanation for this inconsistency proposed by Kemp and Guastella ${ }^{8}$ is that the motivational direction (approach vs. withdrawal) can be confounded by the affective valence (positive vs. negative emotion). In their social-approach/ withdrawal hypothesis, they propose that OT may serve to in-

Received: July 9, 2013 Revised: September 13, 2013

Accepted: September 14, 2013 Available online: April 11, 2014

$\triangle$ Correspondence: Youl-Ri Kim, MD, PhD

Department of Neuropsychiatry, Seoul-Paik Hospital, Inje University, 9 Mareunnae-ro, Jung-gu, Seoul 100-032, Republic of Korea

Tel: +82-2-2270-0063, Fax: +82-2-2270-0344, E-mail: youlri.kim@paik.ac.kr

(a) This is an Open Access article distributed under the terms of the Creative Commons Attribution Non-Commercial License (http://creativecommons.org/licenses/by$\mathrm{nc} / 3.0$ ) which permits unrestricted non-commercial use, distribution, and reproduction in any medium, provided the original work is properly cited. crease approach-related social behaviors while inhibiting withdrawal-related social behaviors.

The synthesis of OT is mainly confined to highly localized nuclei in the rostral hypothalamus of the supraoptic nuclei (SON) and paraventricular nuclei (PVN). However, OT may be distributed in diverse regions of the brain, including the anterior regions of the lateral septum, nucleus accumbens (NAcc), and amygdala. ${ }^{9}$ The region-specific distribution of OT receptors may regulate key aspects of social behavior. The action of OT occurs dynamically through its extracellular diffusion and release into the cerebrospinal fluid (CSF)..$^{10-12}$ OT cannot cross the blood brain barrier (BBB); the difficulty in getting peptides into the human brain has been a major impediment to the study of the effects of neuropeptides on behavior. The intranasal administration of OT is thought to circumvent the BBB, probably by diffusion through the subarachnoid space above the olfactory epithelium. ${ }^{13}$ Intranasal OT has been used to investigate OT circuits within the brain. Intranasal OT is not associated with any adverse outcomes after short-term use in controlled research settings. ${ }^{14}$ The nasal spray can elicit opposing effects in different individuals; hence, a within-subjects experimental design is only capable of investigating the average effect 
pooled across participants. ${ }^{7}$

There is evidence that OT nasal sprays have an effect on the registration of the emotional signal of social information, but there have been some contradictory findings depending on emotional valence. For example, one study reported that OT improved the recognition of happy facial expressions compared to that of negative emotions, ${ }^{15}$ while others reported that negative faces were detected more efficiently than happy ones. ${ }^{16}$ These contradictory findings may be partially due to the differing experimental methods. ${ }^{7}$ The impact of OT administration on standardized experimental methods to measure emotion and behavior may help resolve some of the complexities in understanding social processing. It has been reported that OT reduces threat-related neural activity to socially relevant cues in some subregions of the amygdala. ${ }^{17}$ In a recent study, the mechanism of OT was suggested to modulate the dopamine signals in the ventral tegmental area (VTA), which guides the detection of behaviorally relevant social cues. ${ }^{18}$

The approach-avoidance for emotional experiences construct might be captured by the broader constructs of behavioral activation and inhibition. ${ }^{19,20}$ Evidence from studies designed to assess attentional biases to emotion suggest that the Behavioral Activation System (BAS) predicts vigilant responding to angry faces. ${ }^{21}$ The BAS, as measured by Carver and Whites ${ }^{19}$ the Behavioral Inhibition System and Behavioral Approach System (BIS/BAS) Scale, consists of three subscales: BAS Fun Seeking, BAS Reward Responsiveness, and BAS Drive.

We based our hypotheses on the social approach-withdrawal hypothesis. To prove the social-approach/withdrawal hypothesis of OT, our candidate negative emotions for research are disgust and angry emotions, as these emotions induce an attentional bias of faster detection but also of avoidance. ${ }^{22}$ These two emotions were known not to rate differently for pictures of faces in a study which examined the perceived negative valence of disgust compared to anger. ${ }^{23}$

The aim of study was to examine the role of OT in attentional processes involving social emotions (peers and infants). Firstly, we predicted that OT would reduce the early allocation of selective attention toward negative emotions. Second, we also predicted that OT would increase the allocation of selective attention toward positive emotions.

\section{METHODS}

\section{Participants}

A total of 34 Korean females were recruited in this doubleblind, placebo-controlled, within subject study. The first participant entered the study in October 19, 2012 and the last participant was examined in January 16, 2013. 1 participant were excluded due to low body weight $\left(\mathrm{BMI}=15.82 \mathrm{~kg} / \mathrm{m}^{2}\right)$, and 2 participants withdrawn as they didn't appear in day 2. Therefore a total of 31 participants completed the study.

All of the participants were undergraduate or graduate students who responded to an advertisement posted in the psychology department at a women's university. The age range of the subjects was 19 to 27 years (mean=22.2, SD=2.2), their number of years of education ranged from 12 to 18 (mean $=14.7$, $\mathrm{SD}=1.4$ ), and their IQ ranged from 90 to 134 (mean=115.3, $\mathrm{SD}=10.8$ ). All of the women were nonsmokers, heterosexual, nulliparous, and were not taking any medication including contraceptive pills. The participants completed a brief interview to be screened medical or psychiatric illnesses by a psychiatrist (YK). The participants were assessed using the Korean version of the Wechsler Adult Intelligent Scale. ${ }^{24}$ Exclusion criteria included a self-reported history of major depression, bipolar, panic, or psychotic disorders, substance dependence, epilepsy, eating disorders, and traumatic brain injury. All of the participants were compensated for their travelling expenses and for the time involved in their participation. We tested the participants during the follicular phase of their menstrual cycle (approximately days 4 through 12). This study was a part of the oxytocin project. The whole protocol was approved by both the Korean Food and Drug Association Review (12061) and the Institutional Review Board of Seoul Paik Hospital (IIT-2012-096), and all of the participants provided written informed consent prior to the study. The study was registered the Clinical Research Information Service (http://cris.nih.go.kr) (the registration number: KCT0000716).

\section{Interventions}

\section{OT preparation}

An OT spray was formulated by JW Pharmaceuticals (Korea) from the powder version of the drug (Hemmo Pharmaceuticals, India). The solution was prepared as described previously. ${ }^{15}$ Briefly, $35.2 \mathrm{mg}$ of OT (568 U) was combined with $300 \mathrm{~mL}$ of $0.9 \%$ sodium chloride solution. The $\mathrm{pH}$ was adjusted to 4.01 with $10 \times$ diluted acetic acid. The filtered and sterile solution was distributed in individual vials ( $1.5 \mathrm{~mL}$ each) and stored frozen. On the day of use, the vials were thawed and stored in a refrigerator $\left(4^{\circ} \mathrm{C}\right)$ until required. A clinician prepared the nasal spray by transferring the OT or placebo from the vial into a nebulizer. The nebulizer was primed and given to the participants, who self-administered the nasal spray while being monitored by a clinician and one of the authors. The placebo spray ( $\mathrm{pH} 4.01$ ) was formulated with $0.9 \%$ sodium chloride solution and acetic acid, but without the peptide.

\section{OT application}

OT and the placebo were administered intranasally as de- 
scribed previously ${ }^{4,25}$ with a 1-week interval 45 min before the neuropsychological tasks. At the start of day 1, the participants were asked to self-administer the spray containing either OT or the placebo under the supervision of a researcher. Each participant was randomized in a double-blind manner to either Group 1 (OT first) or to Group 2 (placebo first) (neither the researcher nor the participant knew the group assignment). On day 2, according to the cross-over design, each participant self-administered the spray they had not received on day 1 .

\section{Procedures}

The participants were tested in a private room in Seoul Paik Hospital. They were instructed to abstain from alcohol and caffeine on the day of drug administration and food and drink except water 2 hours before drug administration. Upon their arrival, the participants completed baseline measures of adverse symptoms including abdominal, neurological, dermatological, and cardiac symptoms. Subsequently, the participants self-administered the nasal spray containing OT or the placebo. Several studies using this method have reported OT-dependent effects on behavior and brain function. ${ }^{4}$ Each inhalation of the test solution contained approximately 4 IU of OT. The spray was administered ten times for a total of 40 IU OT. Each administration consisted of one inhalation of the spray into each nostril. The session order of OT and the placebo was randomized using a double-blind procedure. The randomized order of the OT/placebo allocation procedure was generated by the local research supporting system. During the waiting period, the participants completed self-report measures to assess their psychological state including the standardized Korean version of the Beck Depression Inventory ${ }^{26}$ to assess depression, the standardized Korean version of the Spielberger State and the Trait Anxiety Inventory (STAI) ${ }^{27}$ to assess the state and trait anxiety, and the BIS/BAS ${ }^{28}$ to measure personality dimensions which reflect the sensitivity of the motivational systems. The neuropsychological battery of tests began $45 \mathrm{~min}$ after the administration of the spray.

After completing the neuropsychological tests, the participants completed the Positive and Negative Affect Scale (PANAS) ${ }^{29}$ to track their mood changes in positive and negative affect states. Prior to discharge, each participant completed follow-up measures of adverse symptoms. Day 2 testing was identical to day 1 testing, with the only difference being the spray.

\section{Self-rating measures}

\section{Positive and Negative Affect Schedule ${ }^{29}$}

The Positive and Negative Affect Schedule (PANAS) comprises two 10-item mood scales, one assessing positive affect and the other negative affect. As the intranasal OT is delivered and affect shortly, we used the state version of the questionnaire (to assess current affect). In the state version of the questionnaire, the participants are directed to rate "to what extent you feel this way right now; that is, at the present moment". The Korean version of PANAS has been found to have good reliability and validity. ${ }^{30}$

\section{Behavioural Inhibition System and Behavioural Approach System ${ }^{19}$}

The Behavioural Inhibition System and Behavioural Approach System (BIS/BAS) scales measure personality dimensions which reflect the sensitivity of the aversive and appetitive motivational systems (BAS-Drive, BAS-Fun Seeking, and BASReward Responsiveness). The questionnaire contains 24 items written as self-statements on a 4-point forced choice rating scale. The Korean version of BIS/BAS has good internal reliability and reasonable alpha reliability. ${ }^{31}$ Higher scores represent a greater sensitivity in either system.

\section{Visual attention task (Dot probe task) (2,33 $^{32}$}

\section{Stimuli}

The adult faces were obtained from the Korean Facial Expressions of Emotions (KOFEE). ${ }^{34}$ The pool was based on Ekman and Friesen's Facial Action Coding System (1978). ${ }^{35}$ The KOSEF based on the Ekman database of faces has good reliability. ${ }^{34}$ The set used contained 60 images of adults showing 15 positive (happy) or 30 negative (15 angry and 15 disgust) expressions, with each adult having a corresponding picture showing a neutral (15) expression. The picture pair consisted of positive-neutral or negative-neutral expressions of the same adult.

\section{Design and procedure}

Attentional biases were assessed using the dot-probe task. ${ }^{33}$ The dot-probe task based on the Posner paradigm, ${ }^{33}$ which was developed by Macleod ${ }^{32}$ and updated, ${ }^{36,37}$ briefly presents stimuli pairs (e.g., faces) of opposing emotional valence followed by a visual target probe in place of one of the stimuli. The reaction time to recognize the probe is measured as a marker of attention. Faster reactions for probes replacing emotional stimuli relative to those for neutral stimuli indicate attentional vigilance for stimuli of that valence, whilst slower reaction times indicate attentional avoidance. . $^{36,38}$

Each dot-probe trial consisted of a centrally positioned fixation cross for $750 \mathrm{~ms}$, which was replaced by a face-pair displaced at $1000 \mathrm{~ms}$. A probe replaces the location of one of the pictures, on either the left or the right hand side of the screen. Instructions displayed on the screen advised the participants to 
identify as quickly and accurately as possible the probe stimulus which was initially hidden from view. A keyed response from participants ends the trial (Figure 1 for schematic diagram).

The task consisted of 16 practice and 120 experimental trials. In the experimental trials, all combinations of face pairs were used for each model, randomly representing negative (angry, disgust)-neutral, positive (happy)-neutral, and neutral-neutral trials, respectively, making a total of 60 trials, repeated twice. The dot-probe task was presented on E-prime version 2 (Psychology Software Tools, Inc., Pittsburgh, PA, USA).

\section{Statistical analysis}

For the analyses, only the trial reaction times (RTs) for the correct responses were included. The mean RTs for the probes replacing each emotional expression type (negative, neutral or positive) in each block were calculated for each participant whose trials were not missing. Following the procedures used in previous studies, ${ }^{32,39}$ latencies of $<200 \mathrm{~ms}$ and more than 2,000 ms were excluded, as they likely represent lack of attention to the task, and for each participant, outliers were removed by excluding detection latencies that were beyond two standard deviations from their mean (i.e., from each individual's mean RT across all stimuli).

Attentional bias scores were calculated for each trial type (negative-neutral, neutral-neutral and neutral-positive): bias score=PN-PE where $\mathrm{PN}$ is the mean $\mathrm{RT}$ for probes replacing neutral faces and PE is the mean RT for probes replacing emotional (negative or positive) faces. ${ }^{40}$ Therefore, positive scores suggest attentional vigilance towards the emotional stimuli, whilst negative scores indicate attentional bias away from the emotional stimuli. Zero scores suggest no bias towards the emotional face. Initially, biases in the pharmacological challenge (placebo, OT) and emotional valence (positive, negative stimuli) were investigated via a series of initial $2 \times 2$ repeated measures ANOVAs (followed up by paired t-tests). Spearman's rho correlations were used to investigate the relationships between the drug effect on attentional bias and psychological variables. The p-value for two-tailed significance was 0.05 . A Bonferroni correction was applied if multiple testing is used. Analyses were performed using SPSS version 19.

\section{RESULTS}

\section{Characteristics of participants}

The final sample consisted of 31 healthy women. The psychological characteristics of the participants at baseline were as follows; the BDI scores range was from 0 to 29 (mean $=7.07$, $\mathrm{SD}=6.83$ ), the STAI-S scores range was from 23 to 71 (mean= $44.37, \mathrm{SD}=11.30$ ), the STAI-T scores range was from 25 to 74 (mean $=44.37, \mathrm{SD}=11.56)$. The BAS-D score range was from 14 to 21 (mean=18.06, $\mathrm{SD}=2.07$ ), the BAS-FA score range was from 8 to 14 (mean $=10.97, \mathrm{SD}=1.82$ ), the BAS-RR score range was from 12 to 20 (mean=15.77, $S D=2.17$ ), and the BIS score range was from 6 to 15 (mean $=10.65, \mathrm{SD}=2.54)$.

\section{Dot-probe task results}

We conducted a two-way 2 (placebo, OT) $\times 2$ (positive, negative) repeated measures ANOVA. The ANOVA revealed a significant interaction effect between emotions and the drug $[\mathrm{F}(1$, $30)=4.273, \mathrm{p}=0.047$ ] (Figure 2).

In the post hoc paired $\mathrm{t}$-tests between the OT and placebo

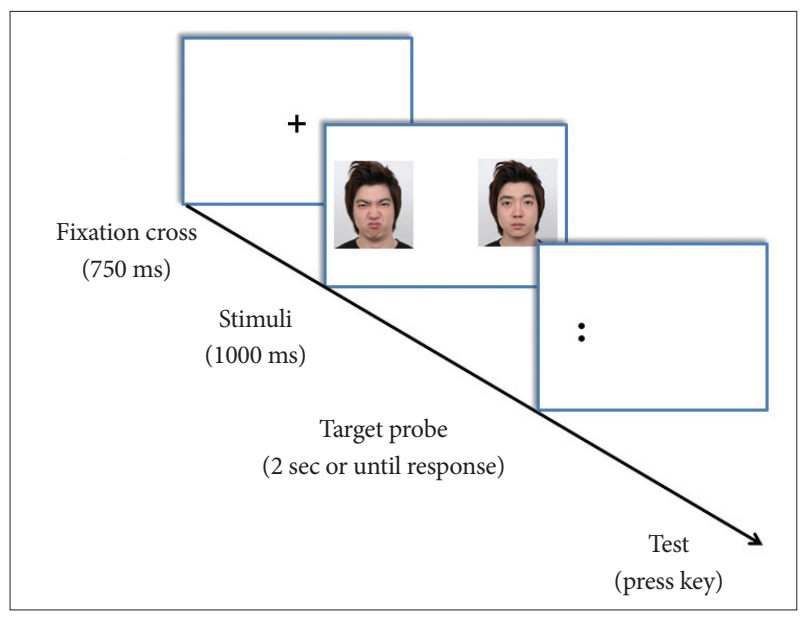

Figure 1. Face dot-probe task paradigm. The dot-probe task briefly presents stimuli pairs (i.e., faces) of opposing emotional valence followed by a visual target probe in place of one of the stimuli. The reaction time to recognize the probe is measured as a marker of attention. Faster reactions for probes replacing emotional stimuli relative to those for neutral stimuli indicate attentional vigilance for stimuli of that valence, whilst slower reaction times indicate attentional avoidance.

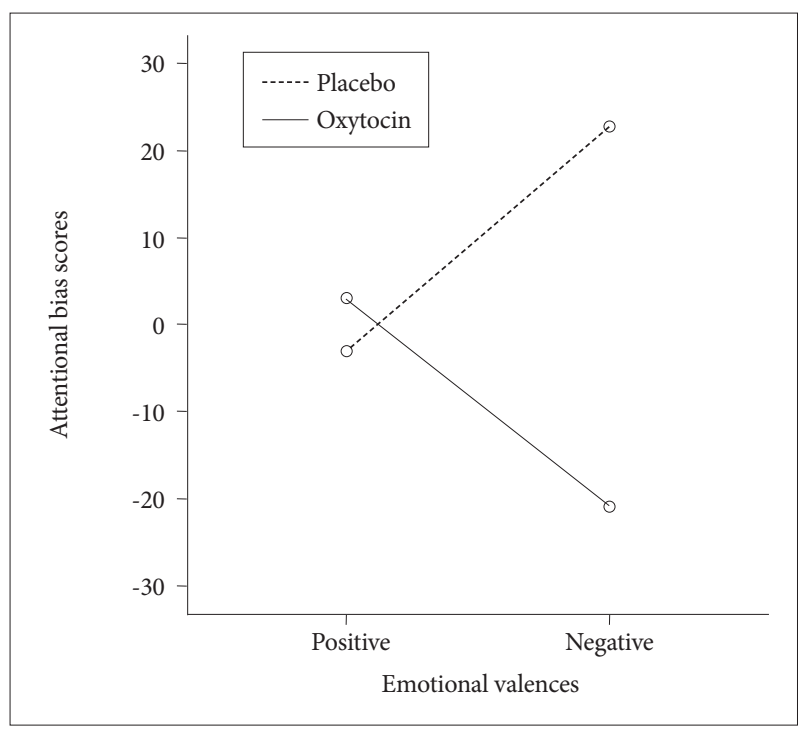

Figure 2. Attentional bias scores for healthy women on face dotprobe task in oxytocin/placebo condition. The interaction effect between drug and emotional valences was $F(1,30)=4.273, p=0.047$. 
conditions, OT specifically reduced the attention bias toward the faces of adults showing negative emotions $(t=3.66, d f=30$, $\mathrm{p}=0.001$ ), and this effect was particularly pronounced in the case of the disgust emotion ( $t=2.694, \mathrm{df}=30, \mathrm{p}=0.011$ ). For the happy faces in the adults' images, the attentional bias in the OT condition was not significantly increased compared with that in the placebo condition. The detailed interference (bias) scores are graphically depicted in Table 1.

\section{Mood after OT/placebo}

The participants had a tendency to report a more positive mood after taking OT than after taking the placebo according to the measured PANAS (placebo: $14.48 \pm 6.78$ vs. OT: 16.35 $\pm 6.37)(\mathrm{t}=2.133, \mathrm{df}=30, \mathrm{p}=0.041)$. No significant differences in negative mood were found between the two conditions (placebo: $11.19 \pm 7.95$ vs. OT: $10.04 \pm 6.95)(t=0.358, d f=30, p=0.723)$ (Figure 3).

We wanted to explore whether the effect of OT was associated with depression, anxiety, anger and motivational approach. Only the BAS-Drive $(r=0.472, \mathrm{p}=0.007)$ was positively correlated with the effect of OT on adult negative emotion. There were no correlations between the effect of OT and the other psychological variables.

\section{DISCUSSION}

The aim of this study was to examine the role of OT in attentional processes regarding social emotions. In this study, healthy women were found to exhibit attentional bias toward negative emotions as compared to positive ones in the placebo condition. We found that OT reduces the selective attention toward the negative emotions of adults. There was no effect of OT on attentional bias to positive emotions in our study. The present data also show that the construct of interest with respect to the effect of OT on withdrawal of attentional bias is the the size of drive measured by the BAS scale.

In the placebo condition, the positive adult faces didn't produce an attentional bias which is consistent with the findings in Caucasians. ${ }^{41}$ On the other hand, negative (anger and disgust) adult faces were associated with increased attention. Our results concerning the attentional bias to negative adult facial expressions in healthy women in the placebo condition are consistent with previous findings that healthy people detected anger and disgust facial expressions more quickly than neutral emotions. ${ }^{22}$ The findings in negative adult faces are somewhat contradictory with other research by our group, ${ }^{41}$ in which avoidance of attentional to negative faces was found but the exposure time differed between these studies $1000 \mathrm{~ms}$ in the current study and 500 and $1250 \mathrm{~ms}$ in Corfield et al. ${ }^{41}$ This technical difference suggests that there may be early vigilance to negative emotions followed by avoidance when later effortful or strategic processing occurs.

In the OT condition, we found that the attentional bias was away from negative adult faces in healthy women, which supports our first hypothesis. The result is consistent with another recent study which found that OT decreased the attention toward sad and angry faces. ${ }^{42}$ We could not find clear evidence in this study that OT influenced the early perception of positive social stimuli of happy faces, contrary to our second hypothesis.



Figure 3. Positive and negative affect states (mean, SD) after oxytocin/placebo. PANAS: positive and negative affect schedule.

Table 1. Attentional bias scores for healthy women on face dot probe task in oxytocin/placebo condition

\begin{tabular}{ccccccc}
\hline Face & Emotion & Oxytocin & Placebo & Difference & T & p-value \\
\hline \multirow{5}{*}{ Adult } & Positive & & & & & \\
& Happy & $2.81(50.66)$ & $-5.84(74.92)$ & $8.65(116.89)$ & 0.434 & 0.668 \\
& Negative & & & & & \\
& Total & $-22.70(50.16)$ & $21.41(46.91)$ & $44.12(669.90)$ & 3.514 & 0.001 \\
& Angry & $-22.51(112.76)$ & $12.12(134.14)$ & $31.05(155.90)$ & 1.123 & 0.270 \\
& Disgust & $-35.28(74.69)$ & $14.16(66.35)$ & $52.27(105.71)$ & 2.694 & 0.011 \\
\hline
\end{tabular}

Data are presented as mean (SD). Because multiple comparisons were made, the Bonferroni corrected significance level was set to alpha $=0.05 / 2(\mathrm{p}<0.025)$ 
Our findings are consistent with Guastella et al. ${ }^{16}$ that angry faces were detected more efficiently than happy faces. In the previous studies for the influence of OT at a top-down stage of information processing, OT enhanced the recognition of positive social memories, ${ }^{6}$ and positive social words. ${ }^{43}$ In this study, we evaluated effects of OT nasal spray at a perceptual bottomup level. Based on the evidence that OT has been shown to influence amygdala reactivity independent of social valence, ${ }^{4}$ Guastella et al. ${ }^{16}$ supposed that OT's evolutionary role may be to reduce the processing of threatening social cues after the initial threat has been detected, and OT may function to enhance positive social cues over social threat when there is opportunity to conceptually process the meaning the cue at later stages of information processing, ${ }^{44,45}$ which can be applicable to our findings.

In our study, the effect of OT on reducing vigilance to adult negative emotion was correlated with the BAS-Drive Our findings are consistent with a previous report that the BAS-Drive and heightened anger are predictors for a vigilant response to angry faces. ${ }^{21}$ These findings on the impact of OT on negative emotion support the social approach-withdrawal hypothesis of intranasal OT.

In our study, the change in the subjective affective state after its administration, suggests that OT may increase positive emotion. A recent review reported that accumulative evidence from clinical trials show that the short-term use of intranasal OT administered to humans, both in males and females, in dosage amounts of up to $40 \mathrm{IU}$ (per dose), resulted in no detectable subjective changes in controlled research settings. ${ }^{46}$ It needs to be considered that our dose of 40 IU of OT is relatively higher than the usual trial dose of 24 IU and there is less previous research in females. Therefore, the question of whether there is a dose-related response to the effect of OT needs to be examined in a future study.

The present findings need to be considered in the context of the following limitations. The first one is we tested with the presenting time of $1000 \mathrm{~ms}$ only in dot probe task, the results of which may differ from early $(<250 \mathrm{~ms})$ or late allocation $(>1250$ $\mathrm{ms}$ ) of selective attention. The other methodological limitation is that we did not test the other negative emotions than angry and disgust, such as sad or fear. So more valences of emotion need to be tested to confirm the effect of OT on attentional bias in negative emotion.

In conclusion, the findings of this study support the hypothesis that OT serves to inhibit vigilance to negative emotions in adults which may reduce withdrawal related social behavior in young women. In a future study, the underlying biological and genetic mechanism underlying the effect of OT on social emotional behaviors needs to be explored.

\section{Acknowledgments}

We thank to Professor Suk Kyoon An for offering adult photos. This study was supported by the Basic Science Research Program through the National Research Foundation of Korea (NRF), which is funded by the Ministry of Education (MOE) (NRF-2011-0024415) to Youl-Ri Kim. Janet Treasure is part funded by the National Institute for Health Research (NIHR) Mental Health Biomedical Research Centre at South London and Maudsley NHS Foundation Trust and King's College London. The views expressed are those of the author(s) and not necessarily those of the NHS, the NIHR or the Department of Health and in addition the Swiss Anorexia Foundation contributed to this work in part the work of Freya Corfield and Janet Treasure.

\section{REFERENCES}

1. Carter CS. Neuroendocrine perspectives on social attachment and love. Psychoneuroendocrinology 1998;23:779-818.

2. Insel TR. Oxytocin-- a neuropeptide for affiliation: evidence from behavioral, receptor autoradiographic and comparative studies. Psychoneuroendocrinology 1992;17:3-35.

3. Shamay-Tsoory SG, Fischer M, Dvash J, Harari H, Perach-Bloom N, Levkovitz Y. Intranasal administration of oxytocin increases envy and schadenfreude (gloating). Biol Psychiatry 2009;66:864-870.

4. Domes G, Heinrichs M, Michel A, Berger C, Herpertz SC. Oxytocin improves "mind-reading" in humans. Biol Psychiatry 2007;61:731-733.

5. Kosfeld M, Heinrichs M, Zak PJ, Fischbacher U, Fehr E. Oxytocin increases trust in humans. Nature 2005;435:673-676.

6. Guastella AJ, Mitchell PB, Mathews F. Oxytocin enhances the encoding of positive social memories in humans. Biol Psychiatry 2008;64: 256-258.

7. Guastella AJ, MacLeod C. A critical review of the influence of oxytocin nasal spray on social cognition in humans: Evidence and future directions. Horm Behav 2012;61:410-418.

8. Kemp AH, Guastella AJ. The role of oxytocin in human affect: a novel hypothesis. Curr Dir Psychol Sci 2011;20:222-231.

9. Bale TL, Davis AM, Auger AP, Dorsa DM, McCarthy MM. CNS region-specific oxytocin receptor expression: importance in regulation of anxiety and sex behavior. J Neurosci 2001;21:2546-2552.

10. Landgraf R, Neumann ID. Vasopressin and oxytocin release within the brain: a dynamic concept of multiple and variable modes of neuropeptide communication. Front Neuroendocrinol 2004;25:150-176.

11. Veening JG, de Jong T, Barendregt HP. Oxytocin-messages via the cerebrospinal fluid: Behavioral effects; a review. Physiol Behav 2010;101: 193-210.

12. Bos PA, Panksepp J, Bluthe RM, van Honk J. Acute effects of steroid hormones and neuropeptides on human social-emotional behavior: a review of single administration studies. Front Neuroendocrinol 2012; 33:17-35.

13. Bahadur S, Pathak K. Physicochemical and physiological considerations for efficient nose-to-brain targeting. Expert Opin Drug Deliv 2012;9:19-31.

14. MacDonald E, Dadds MR, Brennan JL, Williams K, Levy F, Cauchi AJ. A review of safety, side-effects and subjective reactions to intranasal oxytocin in human research. Psychoneuroendocrinology 2011;36:1114-1126.

15. Marsh AA, Yu HH, Pine DS, Blair RJ. Oxytocin improves specific recognition of positive facial expressions. Psychopharmacology (Berl) 2010;209:225-232.

16. Guastella AJ, Carson DS, Dadds MR, Mitchell PB, Cox RE. Does oxytocin influence the early detection of angry and happy faces? Psychoneuroendocrinology 2009;34:220-225.

17. Gamer M, Zurowski B, Buchel C. Different amygdala subregions mediate valence-related and attentional effects of oxytocin in humans. Proc Natl Acad Sci U S A 2010;107:9400-9405.

18. Groppe S, Gossen A, Rademacher L, Hahn A, Westphal L, Gründer G, et al. Oxytocin influences processing of socially relevant cues in the ventral tegmental area of the human brain. Biol Psychiatry 2013;74: 
172-179.

19. Carver CS, White TL. Behavioral-inhibition, behavioral activation, and affective responses to impending reward and punishment-The BIS BAS scales. J Pers Soc Psychol 1994;67:319-333.

20. Gray JA. Issues in the Neuropsychology of Anxiety. Hillsdale, NJ: Erlbaum; 1982.

21. Putman P, Hermans E, van Honk J. Emotional Stroop performance for masked angry faces: it's BAS, not BIS. Emotion 2004;4:305-311.

22. Gilboa-Schechtman E, Foa EB, Amir N. Attentional biases for facial expressions in social phobia: the face-in-the-crowd paradigm. Cogn Emot 1999;13:305-318.

23. Amir N, Najmi S, Bomyea J, Burns M. Disgust and anger in social anxiety. Int J Cogn Ther 2010;3:3-10.

24. Yum TH, Park YS, Oh KJ, Kim JG, Lee HY. The Manual of KoreanWechsler Adult Intelligence Scale. Seoul: Korean Guidance Press; 1992.

25. Born J, Lange T, Kern W, McGregor GP, Bickel U, Fehm HL. Sniffing neuropeptides: a transnasal approach to the human brain. Nat Neurosci 2002;5:514-516.

26. Beck AT, Rush AJ, Shaw BF, Emery G. Cognitive Therapy of Depression: A Treatment Manual. New York: Guilford Press; 1979.

27. Spielberger C, Gorsuch R, Lushene R, Vagg P, Facobs G. Manual for the State-Trait Anxiety Inventory, STAI (Form Y). Palo Alto: Consulting Psychologists Press; 1983.

28. Carver CS, White TL. Behavioral inhibition, behavioral activation, and affective responses to impending reward and punishment: The BIS/ BAS Scales. J Pers Soc Psychol 1994;67:319-333.

29. Watson D, Clark LA, Tellegen A. Development and validation of brief measures of positive and negative affect - the PANAS scales. J Pers Soc Psychol 1988;54:1063-1070.

30. Lim YJ, Yu BH, Kim DK, Kim JH. The positive and negative affect schedule: Psychometric properties of the Korean version. Psychiatry Investig 2010;7:163-169.

31. Kim K, Kim W. Korean BAS/BIS Scale. Korean J Health Psychol 2001; 6:19-37.

32. Macleod C, Mathews A, Tata P. Attentional bias in emotional disorders. J Abnorm Psychol 1986;95:15-20.

33. Posner MI, Snyder CR, Davidson BJ. Attention and the detection of signals. J Exp Psychol 1980;109:160-174.

34. Park JY, Oh JM, Kim SY, Lee MK, Lee CR, Kim BR, et al. Korean Fa- cial Expressions of Emotion (KOFEE). Section of Affect \& Neuroscience, Institute of Behavioral Science in Medicine, Yonsei University College of Medicine, Seoul; 2011.

35. Ekman P, Friesen WV. Facial Action Coding System: A Technique for the Measurement of Facial Movement. Palo Alto, CA: Consulting Psychologists Press; 1978.

36. Koster EH, Crombez G, Verschuere B, De Houwer J. Selective attention to threat in the dot probe paradigm: differentiating vigilance and difficulty to disengage. Behav Res Ther 2004;42:1183-1192.

37. Mogg K, Bradley BP, DeBono J, Painter M. Time course of attentional bias for threat information in non-clinical anxiety. Behav Res Ther 1997; 35:297-303

38. Cisler JM, Bacon AK, Williams NL. Phenomenological characteristics of attentional biases towards threat: a critical review. Cognit Ther Res 2009;33:221-234.

39. MacLeod C, Rutherford E, Campbell L, Ebsworthy G, Holker L. Selective attention and emotional vulnerability: assessing the causal basis of their association through the experimental manipulation of attentional bias. J Abnorm Psychol 2002;111:107-123.

40. Macleod C, Mathews A. Anxiety and the allocation of attention to threat. Q J Exp Psychol A 1988;40:653-670.

41. Corfield F, Cardi V, Leppanen J, Rhind C, Deriziotis S, Hadjimichalis A, et al. Elicited facial expression, emotional experience and attention to infant cues in anorexia nervosa. Unpublished data.

42. Ellenbogen MA, Linnen AM, Grumet R, Cardoso C, Joober R. The acute effects of intranasal oxytocin on automatic and effortful attentional shifting to emotional faces. Psychophysiology 2012;49:128-137.

43. Unkelbach C, Guastella AJ, Forgas JP. Oxytocin selectively facilitates recognition of positive sex and relationship words. Psychol Sci 2008;19: 1092-1094.

44. Williams JMG, Watts FN, MacLeod C, Mathews A. Cognitive Psychology and Emotional Disorders. Chichester, England: Wiley; 1997.

45. Ohman A. The rote of the amygdala in human fear: Automatic detection of threat. Psychoneuroendocrinology 2005;30:953-958.

46. MacDonald E, Dadds M, Brennan J, Williams K, Levy F, Cauchi A. A review of safety, side-effects and subjective reactions to intranasal oxytocin in human research. Psychoneuroendocrinology 2011;36:11141126. 